\title{
Advanced localization of massive black hole coalescences with LISA
}

\author{
Ryan N Lang and Scott A Hughes \\ Department of Physics and Kavli Institute for Astrophysics and Space Research, \\ Massachusetts Institute of Technology, 77 Massachusetts Avenue, Cambridge, \\ MA 02139, USA \\ E-mail: rlang@mit.edu
}

\begin{abstract}
The coalescence of massive black holes is one of the primary sources of gravitational waves (GWs) for LISA. Measurements of the GWs can localize the source on the sky to an ellipse with a major axis of a few tens of arcminutes to a few degrees, depending on source redshift, and a minor axis which is $2-4$ times smaller. The distance (and thus an approximate redshift) can be determined to better than a per cent for the closest sources we consider, although weak lensing degrades this performance. It will be of great interest to search this three-dimensional 'pixel' for an electromagnetic counterpart to the GW event. The presence of a counterpart allows unique studies which combine electromagnetic and GW information, especially if the counterpart is found prior to final merger of the holes. To understand the feasibility of early counterpart detection, we calculate the evolution of the GW pixel with time. We find that the greatest improvement in pixel size occurs in the final day before merger, when spin precession effects are maximal. The source can be localized to within 10 square degrees as early as a month before merger at $z=1$; for higher redshifts, this accuracy is only possible in the last few days.
\end{abstract}

PACS numbers: 04.80.Nn, 04.30.Db, 04.30.Tv 


\section{Introduction}

One of the most promising sources of gravitational waves (GWs) for LISA is the coalescence of massive black hole (MBH) binaries. Observations show that massive black holes are present in the center of nearly all galaxies at the present time [1,2]. Hierarchical structure formation teaches that these galaxies formed from the mergers of smaller galaxies and their associated dark matter halos [3] 4 . During such mergers, dynamical friction and three-body interactions bring the black holes of each galaxy together, forming a binary. Eventually the binary is close enough that it decays due to gravitational radiation [5]. For $10^{4} M_{\odot}<M<10^{7} M_{\odot}$, these waves lie in the low-frequency $\left(3 \times 10^{-5} \mathrm{~Hz}<f<1 \mathrm{~Hz}\right)$ band of LISA. Merger tree calculations show that $\sim$ tens of events should be detected by LISA each year [6,7].

The GWs detected by LISA encode a great deal of information about the source binary. In general, GWs from MBH binaries are characterized by 17 parameters. Since rapid circularization is likely for comparable mass binaries [8], we consider only circular orbits, reducing the parameter set to 15 . These parameters are the masses of the holes, their spins, the orientation of the binary orbit, the 'merger time' and 'merger phase' (defined by the post-Newtonian approximation; see [9] for details), the position of the binary on the sky, and its luminosity distance. LISA estimates of the sky position and distance, along with the errors in those measurements, define a threedimensional 'pixel' in which the source is expected to be located on the sky. It will be interesting to search this pixel for electromagnetic (visible, radio, $x$-ray) counterparts to the GW source.

It is plausible that no significant electromagnetic activity occurs in conjunction with $\mathrm{MBH}$ coalescence. In this case, one could imagine searching the pixel for a galaxy with a structure that indicates a recent merger. Another possibility is to search for a galaxy which has a bulge radial velocity consistent with the GW-measured final black hole mass. (This of course assumes that the well-known relation between these quantities in the local universe [10, 11] holds at high redshift and so soon after a merger.)

It is likely, however, that there is some unique EM activity associated with the $\mathrm{MBH}$ coalescence. The nature of such activity has become a hot research topic in recent years, leading to many possible scenarios [12,13. For example, if the surrounding gas is completely swept away by the binary, there may be no signal during the coalescence. Instead, there would be a delayed afterglow when the gas later accretes onto the remnant hole 14. This afterglow may occur years after the merger. It is likely, though, that the gas will not be totally swept away, leaving enough to accrete onto the holes and create variable EM activity during the inspiral phase. For example, Armitage and Natarajan showed that for a large mass ratio binary, any gas which does remain will be driven in during inspiral, producing an EM signal [15]. More recent work by MacFadyen and Milosavljević showed that periodic variations in the Newtonian potential can create a quasi-periodic EM flux [16].

Other scenarios predict transients during or immediately after the coalescence. One example relies on the fact that the mass of the black holes is partially radiated away by the gravitational waves. This causes a near-instantaneous change in the gravitational potential to which the gas will quickly react, producing shocks [17. Another possibility is that the 'kick', or momentum imparted to the black hole due to an asymmetric emission of GWs [18 20] will send the remnant through the surrounding gas, again producing shocks [21 23]. A transient signal might also appear when the 
GWs are viscously dissipated in the surrounding gas [24].

Finding a counterpart could greatly enhance the science return of $\mathrm{MBH}$ measurement. For example, counterparts can improve LISA's ability to determine certain parameter values. The sky position is correlated with various other parameters, particularly luminosity distance and orbit orientation. When it is determined exactly by identification of a counterpart, the other parameters can be estimated to greater accuracy [25,26]. Another difficulty with parameter estimation is that the estimated masses are actually 'redshifted'; that is, they are equal to the rest frame mass multiplied by $(1+z)$. The GWs themselves only give the luminosity distance, not the redshift, so any decoupling of mass and redshift will be dependent on a cosmological model [25]. The counterpart gives the redshift directly.

Finding the redshift from a counterpart may also allow us to use MBH events as cosmological distance measures. Combining the EM-measured redshift with the GW-measured luminosity distance creates a Hubble diagram which is calibrated only by general relativity [26, 27. In reality, such 'standard sirens $\mathbf{t}$ are affected by weak lensing of the gravitational waves, which impacts measurements of the luminosity distance 28, 29. Still, the systematics affecting MBH GWs should be completely different from those affecting Type Ia supernova standard candles 30 32 and could serve as a useful complement to those sources.

Counterparts are also useful for studying the astrophysics of the MBH coalescence; indeed, the sheer variety of counterpart scenarios shows how uncertain these processes are. Specifically, counterparts may give insight into gas dynamics and accretion. For instance, GW measurements of the mass and EM measurements of the luminosity can be combined to find the Eddington ratio, $L / L_{\text {Edd }}[33$. Finally, the counterparts could be used to test fundamental physics. If a counterpart features EM variation in phase with the gravitational wave signal, the two signals can be compared to test the equivalence of photon and graviton propagation speed. Any difference could be explained by a nonzero graviton rest mass 13 .

Given the potential utility of electromagnetic counterparts, it is important to understand how well LISA will be able to measure sky position and distance. This will give astronomers some indication of LISA's capabilities, as well as provide guidance regarding LISA mission design decisions. In particular, it is interesting to see how well sky position and distance can be measured in advance of merger [34]. The intention is to analyze LISA data in real time so that astronomers can point telescopes at the pixel to look for and then observe the source. Waiting until after the merger means that astronomers will miss any precursor or prompt EM events, which might also make it harder to even identify the correct source.

\section{Preliminaries}

To estimate parameter measurement accuracies, we use the Fisher matrix formalism [35, 36]; details for our analysis are given in [9. The Fisher matrix is defined as

$$
\Gamma_{a b}=\left(\frac{\partial h}{\partial \theta^{a}} \mid \frac{\partial h}{\partial \theta^{b}}\right)
$$

$\ddagger$ This term, coined by Sterl Phinney and Sean Carroll, is appropriate because GWs can be thought of as analogous to sound waves. 
where $h$ is the GW signal measured by LISA and $\theta^{a}$ are the 15 parameters which characterize the signal. The inner product is defined by

$$
(a \mid b)=4 \operatorname{Re}\left[\int_{0}^{\infty} d f \frac{\tilde{a}^{*}(f) \tilde{b}(f)}{S_{n}(f)}\right] .
$$

Tildes denote Fourier transforms, which we take using the stationary phase approximation [36, 37]. $S_{n}(f)$ is the one-sided noise power spectral density, which includes instrument noise [38] and confusion noise from Galactic [39] and extragalactic 40] white dwarf binaries. The inverse of the Fisher matrix is the covariance matrix, $\Sigma^{a b}$, the diagonal entries of which correspond to (squared) parameter errors. The Fisher matrix estimates errors in the 'Gaussian approximation', in which the parameter posterior probability density is represented by a Gaussian around the most likely values. This approximation is known to be good for high signal-to-noise ratio (SNR) [35, 36]. For $z=5$, our signals have median SNR $\sim 10$ a month before merger and $\sim 55-370$ at merger, depending on the black hole masses. For $z=1$, this improves to $\sim 60-95$ a month before merger and $\sim 650-2700$ at merger. However, it is not immediately clear if these values are large enough for a signal with 15 strongly coupled parameters.

We use only the inspiral portion of the coalescence waveform. This is largely because the inspiral can be modeled by the post-Newtonian approximation to general relativity [41 43], offering a convenient parameterization for our analysis. In addition, the primary source of information about sky position is the motion of LISA around the Sun. Therefore, one might guess that the inspiral provides the bulk of the localization. However, with the development of numerical relativity, it has become possible to perform similar calculations which include the merger phase of the waveform. Preliminary results seem to show that the merger could dramatically improve sky position and distance determination [44,45].

Our waveforms are carried out to second post-Newtonian $(2 \mathrm{PN})$ order in the phase, but include only the Newtonian quadrupole amplitude term. They also include the effects of spin-induced precession. Spins precess due to post-Newtonian spin-orbit and spin-spin coupling effects. For example, to the order we are considering [46, 47,

$$
\dot{\mathbf{S}}_{1}=\frac{1}{r^{3}}\left[\left(2+\frac{3}{2} \frac{m_{2}}{m_{1}}\right) \mu \sqrt{M r} \hat{\mathbf{L}}\right] \times \mathbf{S}_{1}+\frac{1}{r^{3}}\left[\frac{1}{2} \mathbf{S}_{2}-\frac{3}{2}\left(\mathbf{S}_{2} \cdot \hat{\mathbf{L}}\right) \hat{\mathbf{L}}\right] \times \mathbf{S}_{1},
$$

where $m_{1}$ and $m_{2}$ are the black hole masses, $M=m_{1}+m_{2}$ is the total mass, $\mu=m_{1} m_{2} / M$ is the reduced mass, $\mathbf{S}_{1}$ and $\mathbf{S}_{2}$ are the spin vectors, $\hat{\mathbf{L}}$ is the normal to the orbital plane, and $r$ is the orbital separation in harmonic coordinates. On short timescales, total angular momentum is conserved, so the orbital plane precesses to compensate for the spins. The effect of these precessions on the waveform is to create various amplitude and phase modulations. For example, consider the 'polarization amplitude', originally introduced by Cutler [48. It is constructed by adding the two polarizations, weighted by their antenna pattern functions $F^{+}$and $F^{\times}$, in quadrature. Various parts of the resulting GW amplitude (varying by author, but always including the geometrical factors) are then defined to be the polarization amplitude. We use the convention of Vecchio [49]:

$$
A_{\mathrm{pol}, i}=\frac{\sqrt{3}}{2}\left[\left(1+(\hat{\mathbf{L}} \cdot \hat{\mathbf{n}})^{2}\right)^{2} F_{i}^{+}(t)^{2}+4(\hat{\mathbf{L}} \cdot \hat{\mathbf{n}})^{2} F_{i}^{\times}(t)^{2}\right]^{1 / 2},
$$


where $\hat{\mathbf{n}}$ is the direction to the binary and $i \in\{\mathrm{I}, \mathrm{II}\}$ denotes one of two detectors synthesized from the LISA laser links. Because $\hat{\mathbf{L}}$ precesses, an amplitude modulation is imposed on the measured waveform. Other modulations are hidden inside the antenna pattern functions.

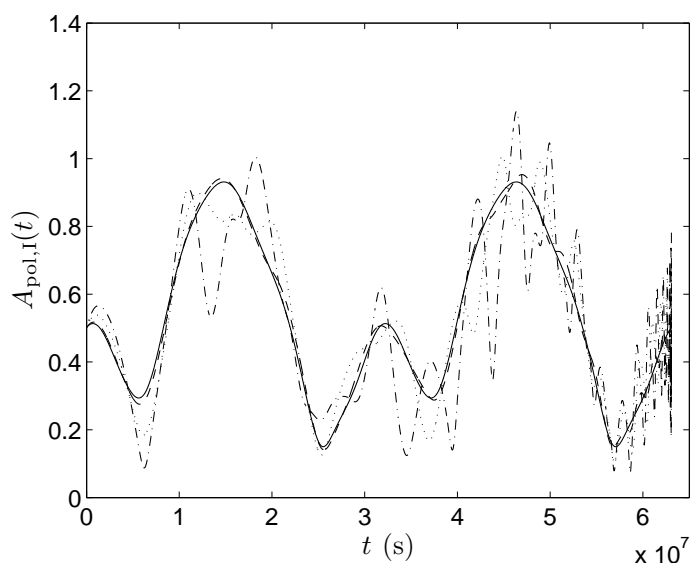

Figure 1. 'Polarization amplitude' $A_{\mathrm{pol}}(t)$ of the signal measured in detector I for a binary with $m_{1}=10^{6} M_{\odot}, m_{2}=3 \times 10^{5} M_{\odot}$, and $z=1$. The curves are as follows: solid line, $\chi_{1}=\chi_{2}=0$; dashed line $\chi_{1}=\chi_{2}=0.1$; dotted line, $\chi_{1}=\chi_{2}=0.5$; and dash-dotted line, $\chi_{1}=\chi_{2}=0.9 . \quad\left(\chi=S / m^{2}\right.$ is the dimensionless spin parameter.) The figure covers the final two years of inspiral. Without spin, the only modulation is due to LISA's orbit around the Sun, which is the primary source of localization information. Precession adds additional modulations, which increase toward merger. (This figure appeared previously as figure 1 of [9] however, an error in the code [50] caused it to be incorrect in the published version.)

Figure 1 shows $A_{\text {pol, } i}$ for a two-year cycle. Without precession, the polarization amplitude has a period of one year, the time it takes LISA to orbit the Sun. This orbital modulation (which is hidden in the pattern functions $F^{+}$and $F^{\times}$) plays a large role in resolving the position of a source on the sky. When precession is added, the amplitude is modulated much more strongly. The additional modulation is strongest just before merger, when the holes are closer and the post-Newtonian precession effects the largest. Similar modulations exist in the relative phase between weighted polarizations (the 'polarization phase') and the intrinsic post-Newtonian phase. Finally, there is an additional precessional correction to the orbital phase [46]. All of these effects combine to provide more information about the source, reducing parameter errors. Specifically, they break degeneracies between strongly correlated parameters, notably the sky position, distance and orbital orientation. We expect the improvements to be greatest when the precession effects are largest, at the end of inspiral.

\section{Time evolution of LISA pixel}

The operation of our code is described in [9]. We first select rest frame masses and redshift for a binary system. We then randomly choose the spin magnitudes and 
direction, orientation, sky position and post-Newtonian 'merger time' parameter $t_{c}$. For each choice, the code computes the Fisher matrix $\Gamma_{a b}$ and the covariance matrix $\Sigma^{a b}$. Extracting the sky position error ellipse from this requires some care. Defining the ellipse such that the probability of the source lying outside it is $e^{-1}(\sim 63 \%$ confidence interval) [48, the major $(2 a)$ and minor $(2 b)$ axes of that ellipse are given by

$$
\begin{aligned}
& \left\{\begin{array}{l}
2 a \\
2 b
\end{array}\right\}=2\left[\csc ^{2} \bar{\theta}_{N} \Sigma^{\bar{\mu}_{N} \bar{\mu}_{N}}+\sin ^{2} \bar{\theta}_{N} \Sigma^{\bar{\phi}_{N} \bar{\phi}_{N}}\right.
\end{aligned}
$$

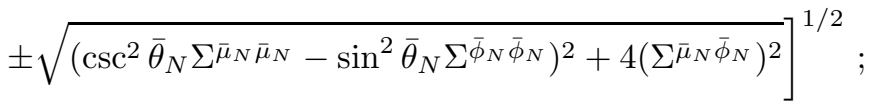

the upper sign is for $2 a$, while the lower sign is for $2 b$. Here $\bar{\mu}_{N}=\cos \bar{\theta}_{N}$, where $\bar{\theta}_{N}$ is the polar angle of the source. $\bar{\phi}_{N}$ is the azimuthal angle. The bars over the angles reflect that they are measured in the barycenter frame, rather than in a frame moving with LISA. (See [9] for more details.)

We find that at merger, the sky position error ellipse has a (median) major axis of $\sim 15-45$ arcminutes at $z=1$, depending on system mass. This figure increases to $\sim 3-5$ degrees at $z=5$. The minor axis ranges from $\sim 5-20$ arcminutes at $z=1$ to $\sim 1-3$ degrees at $z=5$. Finally, the luminosity distance errors $\Delta D_{L} / D_{L}$ are $\sim 0.002-0.007$ at $z=1$ and $\sim 0.025-0.05$ at $z=5$. It is important to note that these luminosity distance errors do not include the effects of weak lensing. Lensing due to intervening matter will magnify or demagnify the waves, causing an incorrect measurement of luminosity distance. The distance error scales with redshift roughly as $\Delta D_{L} / D_{L} \simeq 0.044 z$ for low $z$ [29. It is expected that this dependence will become flat at some transition redshift, most likely near $z \sim 3$ or 4 ; the precise transition depends upon the (poorly understood) high redshift mass function. With the development of high-quality weak lensing maps, one might think that it would be possible to correct for the impact of lensing and recover much of the intrinsic GW distance measurement precision. Unfortunately, lensing noise arises mostly from structure on subarcminute scales that is not probed by shear maps, making any substantial correction impossible 28 .

A complete listing of the time-dependent results can be found in [34]. To help summarize these results, we take an area of 10 square degrees as a useful benchmark for a 'good' localization in the plane of the sky. This is roughly the field of view of the Large Synoptic Survey Telescope (LSST) [51. Then for low redshift $(z=1)$, sources are well localized as far back as a month before merger for most masses. At $z=3$, this condition is met a few days before merger, but only for a subset of the masses we consider. Finally, at $z=5$, the error ellipse is the size of the LSST field or smaller at most a day before merger, and only then in very few cases.

At $z=1$, the luminosity distance errors increase to $\sim 2-4 \%$ a month before merger in most cases. Lensing should dominate the error budget for all but the highest masses, for which the source is in band too short a time to be well localized (if it is in band yet at all). At $z=3$, the distance errors are as large as $\sim 10 \%$ or more a month before merger; $\sim 20 \%$ becomes more typical at $z=5$. Since the lensing error flattens out with redshift, these intrinsic errors might dominate, but the high- $z$ lensing noise is too uncertain to make a definite statement.

Figure 2 shows the evolution of the major axis and minor axis of the sky position error ellipse for $m_{1}=10^{6} M_{\odot}, m_{2}=10^{5} M_{\odot}$ and $z=1$. Each histogram represents $10^{4}$ 

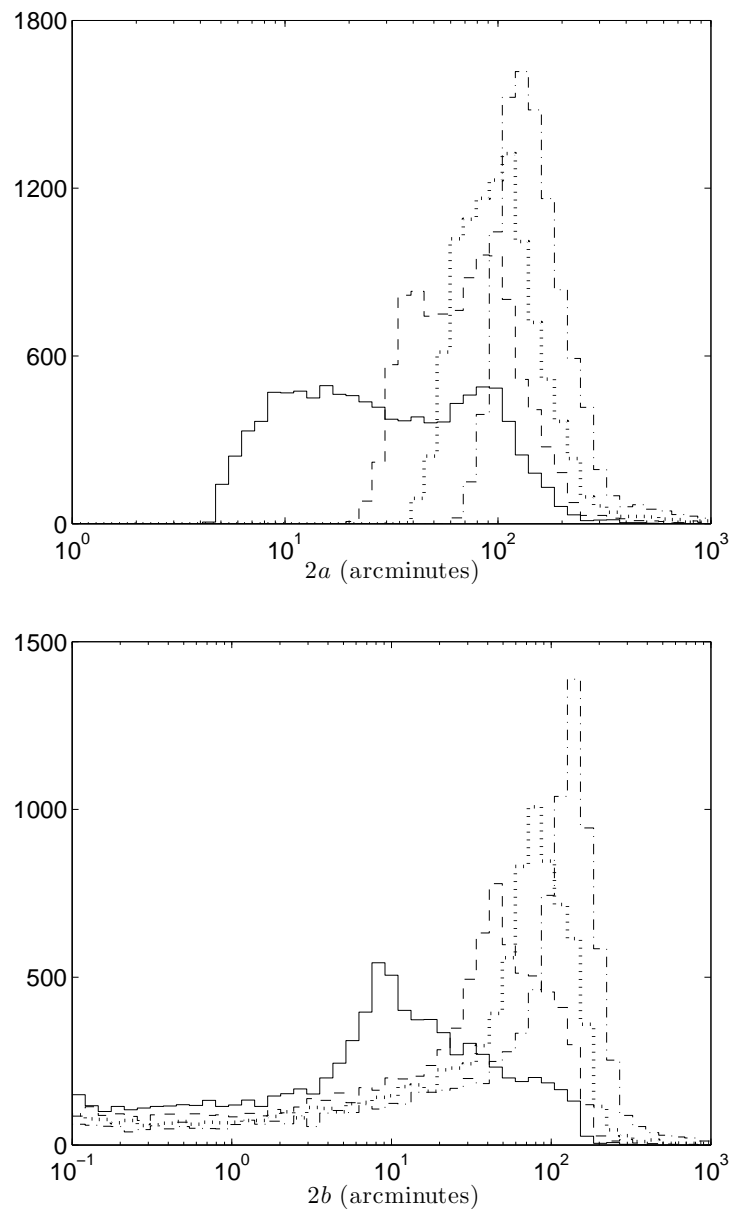

Figure 2. Distribution of the major axis $2 a$ (top) and minor axis $2 b$ (bottom) of the sky position error ellipse for $10^{4}$ binaries with $m_{1}=10^{6} M_{\odot}, m_{2}=10^{5} M_{\odot}$, and $z=1$ at different times before merger. Reading from left to right, the number of days until merger is 0 (solid line), 1 (dashed line), 7 (dotted line), and 28 (dash-dotted line). The largest change occurs in the final day before merger.

different choices of the random parameters. As the time until final merger decreases from 1 month to 1 day, the median error gradually decreases. In addition, the shape of the distribution changes slightly, becoming less peaked. However, a drastic change occurs between 1 day and 0 days (end of inspiral). In just this day, the median is reduced by half an order of magnitude and the shape of the distribution changes drastically. For the major axis, the distribution becomes almost flat over a certain range and displays a slightly bimodal structure.

Figure 3 shows the median values of $2 a$ for different masses. We see a trend holding over all masses: gradual improvement with time until a sharp change the last day. The change is greatest for the lowest mass systems. These results agree quite well with those of Kocsis et al [52, except their study does not show a sharp improvement in localization during the final day of inspiral. It turns out that this effect is due 

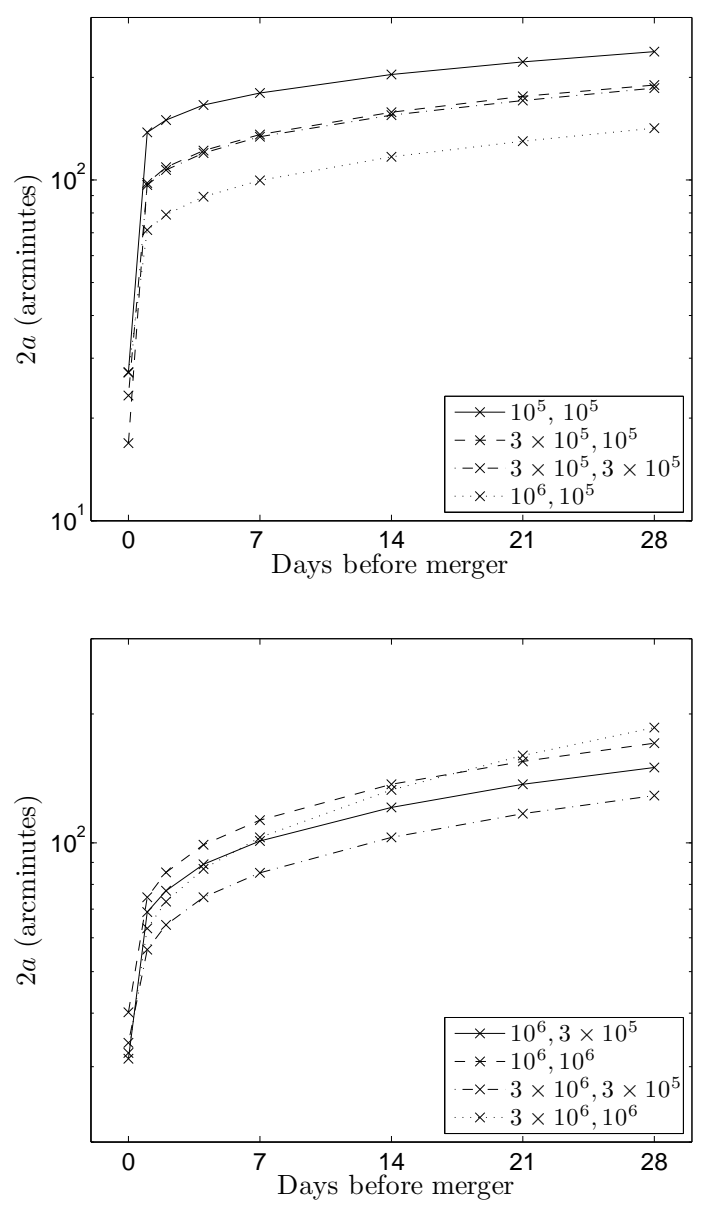

Figure 3. Median values of $2 a$, the sky position ellipse major axis, as a function of time before merger. Each point is taken from a Monte Carlo sample of $10^{4}$ binaries with fixed masses (given in units of $M_{\odot}$ ) and $z=1$. Data were only output at the marked points; the lines are there to guide the eye.

mostly to spin precession, which Kocsis et al do not include. This late impact of precession is to be expected following our discussion of figure 1 and is confirmed in figure 4. By breaking correlations between parameters, precession gives a factor of $2-7$ improvement in final parameter estimation errors. However, since most of this improvement takes place in the final days before merger, precession does not help very much with advanced localization. This effect may, however, impact the frequency of data transfer between LISA and the ground: as a source approaches merger, it may be useful to continuously downlink data to take advantage of the rapid improvement in localization.

As a final study, we also investigate the position dependence of the localization pixel. Rather than randomly choosing the full sky position, we randomly choose just one of the polar $\left(\bar{\theta}_{N}\right)$ and azimuthal $\left(\bar{\phi}_{N}\right)$ angles, while varying the other in a 


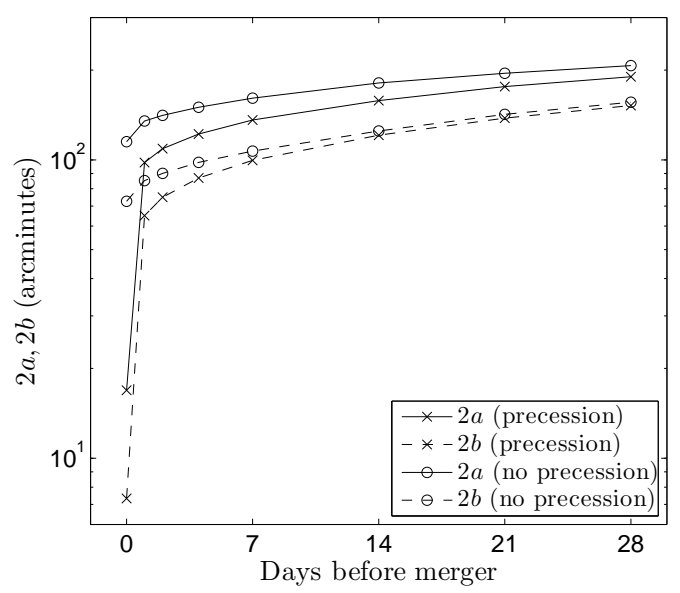

Figure 4. Medians of $2 a$ (solid lines) and $2 b$ (dashed lines) as a function of time for binaries with $m_{1}=3 \times 10^{5} M_{\odot}, m_{2}=10^{5} M_{\odot}$ and $z=1$. Spin-induced precession was included in the waveform for the crosses and neglected for the circles.

controlled fashion. It turns out that only the polar dependence is meaningful due to a particular feature of our formalism: we randomly choose the final merger time within LISA's mission window, meaning that even with a specifically chosen $\bar{\phi}_{N}$, the relative azimuth between LISA and the source at merger, $\delta \phi=\bar{\phi}_{N}-\phi_{\text {LISA }}\left(t_{c}\right)$, is random. This mostly washes out the azimuthal dependence. Other authors [13,52,53, make a different choice, in which the position of LISA at merger is fixed. This allows them to study the position dependence of localization at a particular time of the year; consequently, their results for both polar and azimuthal angle dependence are different from ours.

We find that the sky position axes and area are maximal for sources in the ecliptic plane and minimal near the poles, at $\cos \bar{\theta}_{N}= \pm 0.8$. The distance errors have a threepeaked structure, with one peak at the ecliptic plane and the others very close to where the sky position errors are minimized. Converting to Galactic coordinates, we find that some of the best localized sources lie outside the Galactic plane. This suggests that the most easily localized binaries will not tend to lie in regions of the sky with thick foregrounds or high levels of extinction.

\section{Summary and future work}

Observing electromagnetic counterparts to massive black hole binary coalescences can be very useful in the contexts of parameter estimation, cosmology, astrophysics and fundamental physics. To observe a counterpart, and to get the most science out of it, it is useful to be able to localize the source prior to merger. Our study shows that advanced localization of sources with LISA should be possible at low redshift $(z=1)$, but begins to be difficult at higher $z$. The effects of precession break degeneracies between parameters, dramatically improving estimates in the final days before merger. Finally, at least some of the best located sources will be out of the Galactic plane, 
improving our chances of identifying counterparts.

These results are not the final story on LISA's ability to measure and localize MBH sources. For example, it is useful to test whether the Gaussian approximation/Fisher matrix approach is actually valid for a signal with 15 parameters. This problem is currently being approached both by analytic analysis [54] and by comparing the Fisher results to errors generated by a Markov Chain Monte Carlo exploration of the parameter posterior distribution function [55]. Another approximation worth checking is the stationary phase method for obtaining Fourier transforms; this is known to be good for signals without precession 56 but seems to smooth out sharp features caused by strong precession [57]. Finally, there are various extra pieces which can still be added to the waveform, including higher post-Newtonian phase terms, higher order precession equations [58,59, higher postNewtonian amplitude terms, and harmonics beyond the quadrupole. The latter two terms, often called simply 'higher harmonics', have already been shown to decrease parameter errors in the absence of spin precession [53, 60, 61. The improvement seems to be somewhat orthogonal to the improvement caused by precession, but a more complete investigation, including time dependence, is necessary. We and our collaborators are currently investigating these issues.

\section{Acknowledgments}

Our work on LISA measurement analysis has been supported by NASA Grants NNG05G105G and NNX08AL42G, as well as NASA contract no. 1291617 and the MIT Class of 1956 Career Development Fund. We thank Daniel Holz for attempting to educate us on the redshift dependence of weak lensing distance errors. We also gratefully thank the organizers of the Symposium for the travel support provided to RNL and for the outstanding meeting that they put together.

\section{References}

[1] Kormendy J and Richstone D 1995 Ann. Rev. Astron. Astrophys. 33581

[2] Magorrian J et al 1998 Astron. J. 1152285

[3] Madau P 2007 Proc. XVIII Canary Islands Winter School of Astrophysics on The Emission Line Universe (Tenerife, 2006) ed J Cepa and F Sanchez (Cambridge, UK: Cambridge University Press) arXiv:0706.0123

[4] Di Matteo T, Colberg J, Springel V, Hernquist L and Sijacki D 2008 Astrophys. J. 67633

[5] Begelman M C, Blandford R D and Rees M J 1980 Nature 287307

[6] Sesana A, Volonteri M and Haardt F 2007 Mon. Not. R. Astron. Soc. 3771711

[7] Micic M, Holley-Bockelmann K, Sigurdsson S and Abel T 2007 Mon. Not. R. Astron. Soc. 380 1533

[8] Peters P C 1964 Phys. Rev. 136 B1224

[9] Lang R N and Hughes S A 2006 Phys. Rev. D 74122001

[10] Ferrarese L and Merritt D 2000 Astrophys. J. Lett. 539 L9

[11] Gebhardt K et al 2000 Astrophys. J. Lett. 539 L13

[12] Dotti M, Salvaterra R, Sesana A, Colpi M and Haardt F 2006 Mon. Not. R. Astron. Soc. 372 869

[13] Kocsis B, Haiman Z and Menou K 2008 Astrophys. J. 684870

[14] Milosavljević M and Phinney E S 2005 Astrophys. J. Lett. 622 L93

[15] Armitage P J and Natarajan P 2002 Astrophys. J. Lett. 567 L9

[16] MacFadyen A I and Milosavljević M 2008 Astrophys. J. 67283

[17] Bode N and Phinney E S 2007 APS Abstract http://meetings.aps.org/link/BAPS.2007.APR.S1.10

[18] Fitchett M J 1983 Mon. Not. R. Astron. Soc. 2031049

[19] Campanelli M, Lousto C O, Zlochower Y and Merritt D 2007 Phys. Rev. Lett. 98231102 
[20] Schnittman J D, Buonanno A, van Meter J R, Baker J G, Boggs W D, Centrella J, Kelly B J and McWilliams S T 2008 Phys. Rev. D 77044031

[21] Lippai Z, Frei Z and Haiman Z 2008 Astrophys. J. Lett. 676 L5

[22] Shields G A and Bonning E W 2008 Astrophys. J. 682758

[23] Schnittman J D and Krolik J H 2008 Astrophys. J. 684835

[24] Kocsis B and Loeb A 2008 Phys. Rev. Lett. 101041101

[25] Hughes S A 2002 Mon. Not. R. Astron. Soc. 331805

[26] Holz D E and Hughes S A 2005 Astrophys. J. 62915

[27] Schutz B F 1986 Nature 323310

[28] Dalal N, Holz D E, Chen X and Frieman J A 2003 Astrophys. J. Lett. 585 L11

[29] Holz D E and Linder E V 2005 Astrophys. J. 631678

[30] Phillips M M 1993 Astrophys. J. Lett. 413 L105

[31] Riess A G, Press W H and Kirshner R P 1995 Astrophys. J. Lett. 438 L17

[32] Wang L, Goldhaber G, Aldering G and Perlmutter S 2003 Astrophys. J. 590994

[33] Kocsis B, Frei Z, Haiman Z and Menou K 2006 Astrophys. J. 63727

[34] Lang R N and Hughes S A 2008 Astrophys. J. 6771184

[35] Finn L S 1992 Phys. Rev. D 465236

[36] Cutler C and Flanagan É É 1994 Phys. Rev. D 492658

[37] Poisson E and Will C M 1995 Phys. Rev. D 52848

[38] Larson S L, Hiscock W A and Hellings R W 2000 Phys. Rev. D 62062001

[39] Nelemans G, Yungelson L R and Portegies Zwart S F 2001 Astron. Astrophys. 375890

[40] Farmer A J and Phinney E S 2003 Mon. Not. R. Astron. Soc. 3461197

[41] Blanchet L 2006 Liv. Rev. Rel. 94

[42] Blanchet L, Damour T, Iyer B R, Will C M and Wiseman A G 1995 Phys. Rev. Lett. 743515

[43] Will C M and Wiseman A G 1996 Phys. Rev. D 544813

[44] Babak S, Hannam M, Husa S and Schutz B 2008 arXiv:0806.1591

[45] Thorpe J I, McWilliams S T, Kelly B J, Fahey R P, Arnaud K and Baker J G 2008 arXiv:0811.0833

[46] Apostolatos T A, Cutler C, Sussman G J and Thorne K S 1994 Phys. Rev. D 496274

[47] Kidder L E 1995 Phys. Rev. D 52821

[48] Cutler C 1998 Phys. Rev. D 577089

[49] Vecchio A 2004 Phys. Rev. D 70042001

[50] Lang R N and Hughes S A 2007 Phys. Rev. D $75089902(\mathrm{E})$

[51] Tyson J A et al 2002 Proc. SPIE 483610

[52] Kocsis B, Haiman Z, Menou K and Frei Z 2007 Phys. Rev. D 76022003

[53] Trias M and Sintes A M 2008 Phys. Rev. D 77024030

[54] Vallisneri M 2008 Phys. Rev. D 77042001

[55] Cornish N J and Crowder J 2005 Phys. Rev. D 72043005

[56] Droz S, Knapp D J, Poisson E and Owen B J 1999 Phys. Rev. D 59124016

[57] Cornish N J, O'Sullivan S, Lang R N and Hughes S A in preparation

[58] Faye G, Blanchet L and Buonanno A 2006 Phys. Rev. D 74104033

[59] Blanchet L, Buonanno A and Faye G 2006 Phys. Rev. D 74104034

[60] Arun K G, Iyer B R, Sathyaprakash B S, Sinha S and Van Den Broeck C 2007 Phys. Rev. D 76 104016

[61] Porter E K and Cornish N J 2008 Phys. Rev. D 78064005 